\title{
Robust Atrial Ectopic Beat Classification From Surface ECG Using Second-Order Blind Source Separation
}

\author{
Yingjing Feng ${ }^{1,2}$, Caroline Roney ${ }^{3}$, Mélèze Hocini ${ }^{4}$, Steven Niederer $^{3}$, Edward Vigmond $^{1,2}$ \\ ${ }^{1}$ IHU Liryc, Electrophysiology and Heart Modeling Institute, fondation Bordeaux Université, \\ Pessac-Bordeaux, France \\ ${ }^{2}$ Univ Bordeaux, IMB, UMR 5251, Talence, France \\ ${ }^{3}$ Division of Imaging Sciences \& Biomedical Engineering, King's College London, London, United \\ Kingdom \\ ${ }^{4}$ Bordeaux University Hospital (CHU), Electrophysiology and Ablation Unit, Pessac, France
}

\begin{abstract}
Ectopic beats (EBs) generated from the atria or pulmonary veins are an important trigger mechanism for atrial fibrillation $(A F)$. They can be periodic, and have been commonly observed during AF episodes. Robust noninvasive detection of EBs could improve pre-operative prediction, as well as post-ablation management. By separating periodic sources from surface ECG using second-order blind source separation methods, EBs were extracted, and discriminated from $A F$ reentries, another type of periodic source. Our method is robust to noise of up to $0.5 \mathrm{mV}$ in the ECG, achieving an area-under curve of receiver operating characteristic (AUC-ROC) of $0.89 \pm 0.01$ over a synthetic dataset of 31 reentries and $58 \mathrm{EBs}$, with and without Acetylcholine modulation in the left atrium.
\end{abstract}

\section{Introduction}

Atrial ectopic beats (EBs) arising from foci are an important mechanism of atrial fibrillation (AF), and have been commonly observed amongst AF patients in their initial episodes, as well as post-ablation. Robust non-invasive detection of EBs could improve the pre-operative planning for pulmonary vein isolation, and the assessment of EBs could be used for post-ablation monitoring.

Surface ECGs can be seen as sensor signals produced by a number of unknown sources. There exist a number of blind source separation (BSS) methods, based on assumptions of inter-source independence, or specific structures within the source. Among them, second-order BSS (SOBSS) methods exploit covariance matrices with optimal linear predictability to extract periodic sources from signals [1]. Unlike most BSS algorithms which require additional steps to estimate the optimal number of sources, they have less restrictions in selecting desirable sources, and the contribution of sources can be ranked. Thus, SO-BSS methods were adopted to extract periodic atrial sources $[2,3]$, assuming AF signals over a short period exhibit quasi-periodicity, and to separate maternal from fetal ECG signals [4], but were never used to distinguish between different atrial sources, such as focal sources or reentries.

This study developed an SO-BSS-based algorithm to analyse the torso ECG to distinguish atrial EBs from reentries, another major kind of periodic source underlying AF.

\section{Methods}

\subsection{Modelling Reentries and EBs}

We modelled spatiotemporally stable reentries and regular EBs in AF, each lasting for $3225 \mathrm{~ms}$ (reentries) or $2000 \mathrm{~ms}$ (EBs), in an anatomically realistic biatrial mesh with the CARPentry simulator [5], and computed their surface potentials. The biatrial mesh was made from a lategadolinium enhanced magnetic resonance imaging scan of an AF patient from the CHU Bordeaux, with the addition of inter-atrial structures, as well as specification of regional eletrophysiological properties as in [6]. We ran monodomain simulations with lead positions shown in Fig. 1 (e-f) and computed standard 12-lead ECGs, 252lead vest, and 2835-lead torso body surface potential maps (BSPMs) for each simulation. The last segments of specified lengths $(600,800, \ldots, 1400 \mathrm{~ms})$ to be used for analysis went through a second-order Butterworth filter with a passband of 2 to $30 \mathrm{~Hz}$ and were subtracted by the Wilson's central terminal. Before inducing AF, we applied five stimuli at the sinoatrial node with an interval of $700 \mathrm{~ms}$ to entrain the atria to sinus rhythm. We computed $\alpha_{L A}, \beta_{L A}, \alpha_{R A}$, and $\beta_{R A}$ of the universal atrial coordinate system [7] to specify locations of conduction blocks (CBs) and foci on the left atrium (LA) and right atrium (RA) (Fig. 1 (a-d)). 


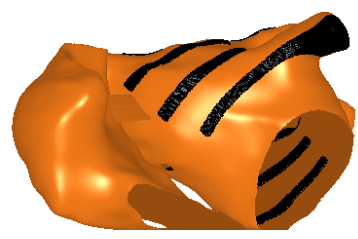

(a)

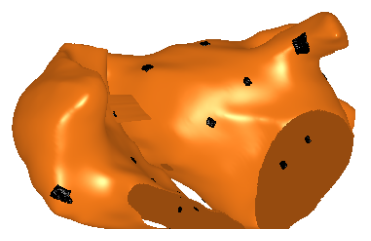

(c)

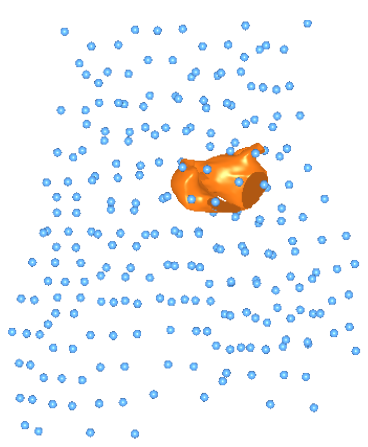

(e)

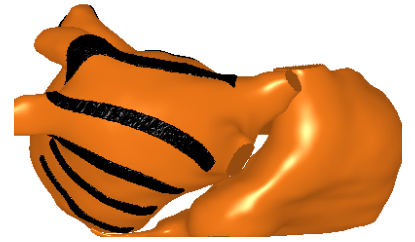

(b)

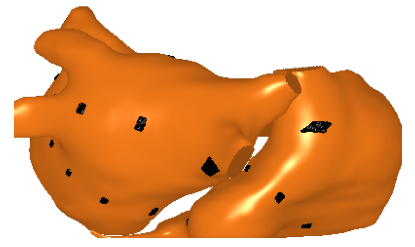

(d)

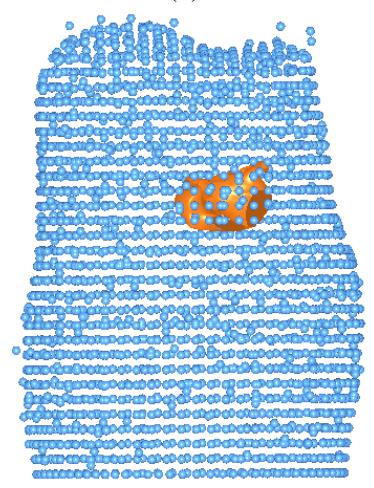

(f)
Fig. 1: Locations for CBs with $\beta_{L A} \in[0.2-0.8]$ (a-b), and foci (c-d). (e) Vest leads. (f) Torso leads.

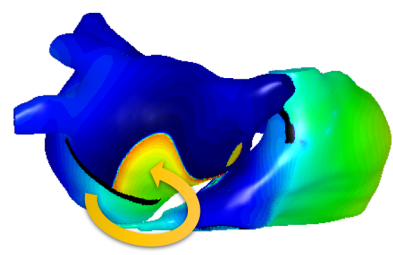

(a) A reentry with a $\mathrm{CB}$

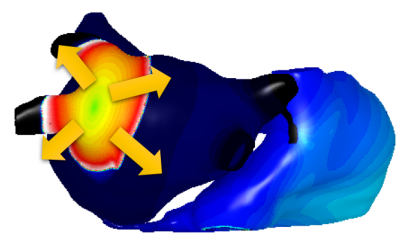

(b) An EB without ACh
$-80 \mathrm{mV}$ $15 \mathrm{mV}$

Fig. 2: The transmembrane voltage of posterior LA for a reentry and an $\mathrm{EB}$ of $\mathrm{AF}$. (a) A reentry is anchored around a CB (black line) where $C B\left(\beta_{L A}\right)=0.2$ and $\alpha_{L A}$ is $0.2-$ 0.8 . (b) An EB originating from $\left(\alpha_{L A}=0.6, \beta_{L A}=0.4\right)$.

A cross-field stimulation was used to initiate reentries (Fig. 2(a)): an S1 stimulus was first applied on an isthmus of $\alpha_{L A}=0$, and after $225 \mathrm{~ms}$, when a third of LA tissue has been repolarised, an $\mathrm{S} 2$ stimulus of $2 \mathrm{~ms}$ was applied on nodes of $\beta_{L A}$ ranging from 0 up to $C B\left(\beta_{L A}\right) \in$ $\{0.1,0.2, \ldots, 0.8\}$. Anatomical CBs were also added on the LA, where $\beta_{L A}$ of each CB corresponded to $C B\left(\beta_{L A}\right)$, and $\alpha_{L A}$ from $0.2-0.8,0.3-0.7,0.4-0.6$ and $0.3-0.5$.
In total there were 31 simulations that maintained stable reentries since $\mathrm{S} 2$ ( $\geq 2000 \mathrm{~ms}$ ), with or without added CBs.

Simulated EBs (Fig. 2(b)) were triggered every $100 \mathrm{~ms}$ at 29 foci of 0.02 length in $\alpha$ and $\beta$ on the LA and RA, centered at combinations of $(\alpha, \beta)$ where $\alpha, \beta \in$ $\{0.2,0.4,0.6,0.8\}$, and 3 foci were discarded for not appearing on the LA or RA surface. To add heterogeneity, we also simulated islands of Acetylcholine (ACh) release $([\mathrm{ACh}]=1 \mu \mathrm{M})$ in the LA and LA appendage using a modified Kneller's model $[8,9]$, wherein the conduction velocity was kept as [6], and 60,80, and $1002 \mathrm{~mm}$ radius $\mathrm{ACh}$ islands were randomly distributed in $\alpha_{L A}$ of $0-0.33,0.33-0.67$, and $0.67-1$, respectively.

\subsection{Classification of EBs with SO-BSS}

To separate the atrial sources, we assumed $M$-lead surface ECGs over the time variable $t$ as $\mathbf{X}(t)=$

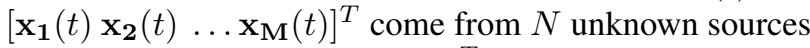
$\mathbf{S}(t)=\left[\begin{array}{llll}\mathbf{s}_{\mathbf{1}}(t) & \mathbf{s}_{\mathbf{2}}(t) & \ldots & \mathbf{s}_{\mathbf{N}}(t)\end{array}\right]^{T}, \mathbf{W}$ is a linear mixing matrix, and $\mathbf{X}_{\mathbf{w}}$ is the pre-whitened matrix of $\mathbf{X}$, such that $\mathbf{X}_{\mathrm{w}}=\mathbf{W S}$. To maximize the periodicity of a source is to minimize the following measure:

$\epsilon(\mathbf{w}, \tau)=\frac{\sum_{t}\left|\mathbf{s}_{i}(t+\tau)-\mathbf{s}_{i}(t)\right|^{2}}{\sum_{t}\left|\mathbf{s}_{i}(t)^{2}\right|}=2\left[1-\frac{\mathbf{w}^{H} C_{x}(\tau) \mathbf{w}}{\mathbf{w}^{H} C_{x}(0) \mathbf{w}}\right]$,

where a $\tau$-lag cumulant matrix was defined as $C_{x}(\tau)=$ $\mathbf{X}(t+\tau) \mathbf{X}(t)^{T}$. The SO-BSS method AMUSE [10] exploits the idea that the vector $\mathbf{w}$ minimizing $\epsilon(\mathbf{w}, \tau)$ is given by the eigenvector corresponding to the largest eigenvalue from the generalized eigenvalue decomposition (GEVD) on the matrix pair $\left(C_{x}(\tau), C_{x}(0)\right)$. The sources could then be recovered with $\tilde{\mathbf{S}}=\tilde{\mathbf{W}}^{H} \mathbf{X}_{\mathbf{w}}$, where $\tilde{\mathbf{W}}^{H}$ is the Hermitian transpose of the matrix containing eigenvectors from the GEVD as columns.

To estimate the time lag $\tau$ in $C_{x}(\tau)$ in AMUSE, we used another SO-BSS method SOBI [11], a generalized version of AMUSE, which replaced GEVD with joint diagonalization (JD) on multiple cumulant matrices with different time lags. For atrial periodic sources (foci and reentries), we calculated 241 cumulant matrices with time lags of $80-320 \mathrm{~ms}$, similar to [2]. After JD, we obtained $N$ eigenvectors and 241 diagonal matrices each containing $N$ eigenvalues corresponding to $N$ sources, and ranked these $N$ sources from high to low according to the maximal eigenvalue of each source.

To assess the $\tau$-lag periodicity of an estimated source $\tilde{\mathbf{s}}_{i}$, we adopted the auto-correlation function (ACF) [12] on $\tilde{\mathbf{s}}_{i}$ :

$$
A C F_{i}(\tau)=\frac{\sum_{t=1}^{T-\tau}\left(\tilde{\mathbf{s}}_{i}(t)-\overline{\mathbf{s}}_{i}\right)\left(\tilde{\mathbf{s}}_{i}(t+\tau)-\overline{\mathbf{s}}_{i}\right)}{\sum_{t=1}^{T}\left(\tilde{\mathbf{s}}_{i}(t)-\overline{\mathbf{s}}_{i}\right)^{2}}
$$

where $\overline{\mathbf{s}}_{i}$ is the expected value of $\tilde{\mathbf{s}}_{i}$. Starting from the top ranked source of SOBI, we searched for the highest peak 
between $80-320 \mathrm{~ms}$ of prominence $\geq 0.4$ following a sink of prominence $\geq 0.4$, and the search went on to the next ranked source until such a peak was identified. The time lag $\tilde{\tau}$ of the resultant peak was then chosen as the periodic cycle of the main source, and was passed to AMUSE to construct $C_{x}(\tilde{\tau})$. The sources separated by AMUSE were ranked from high to low, again, by their corresponding eigenvalues of GEVD.

Finally, to classify EBs, we defined an accumulated $\tilde{\tau}$ lag $\mathrm{ACF}$ (AACF) as $A A C F(K, N)=\sum_{i=1}^{K} A C F_{i}(\tilde{\tau})$, where $K$ and $N$ are the preserved and total number of sources by AMUSE, respectively. The first few sources have, naturally, high $A C F_{i}(\tilde{\tau})$; for signals containing a simple periodic source such as EBs, the lower-ranked sources still preserve a relatively high $A C F_{i}(\tilde{\tau})$. For reentries however, these low-ranked sources are likely to preserve a different period, resulting in a lower $A C F_{i}(\tilde{\tau})$. Our method could be run on any modern computing device as the complexity mainly came from JD.

\section{Results}

We separated 12-lead ECG, 252-lead vest BSPM, and 2835-lead torso BSPM of each simulation into $N$ periodic sources ( $5 \leq N \leq 9$ ), with added Gaussian noises of a standard deviation $\sigma \in\{0,0.1,0.5\} \mathrm{mV}$ on each lead of the signal, representing zero, middle and high-level noises.

Fig. 3 shows sources of a reentry and an EB from an $1000 \mathrm{~ms}$ ECG, in which the lower-ranked sources were more organised in EBs compared to stable reentries. For all the $1000 \mathrm{~ms}$ signals, AUC-ROC scores using $A A C F(K, N)$ are shown in Fig. 4, where the scores in all case converged for $K \geq 4$. Using $A A C F(N, N)$ for classification, the AUC-ROCs for all noise levels and recording systems are $0.89 \pm 0.01$. The comparison with single-lead methods, the power density degree of the dominant frequency (DF), $A F F T r_{2 D F}$ [13], as well as using $\tilde{\tau}$-lag ACF on V1 without performing further BSS, $A C F_{V 1}(\tilde{\tau})$, is shown in Table 1. Pairwise paired t-tests showed no significant difference on AUC-ROCs between different recording systems, choices of $N$, or noise levels, although $N=7$ has the smallest variance of AUC-ROCs.

\begin{tabular}{c|ccc}
\hline Noise $(\sigma, \mathrm{mV})$ & 0 & 0.1 & 0.5 \\
\hline$A F F T r_{2 D F}$ & 0.47 & 0.53 & 0.50 \\
$A C F_{V 1}(\tilde{\tau})$ & 0.82 & 0.56 & 0.49 \\
$\mathbf{A A C F}(\mathbf{N}, \mathbf{N})$ & $\mathbf{0 . 8 9} \pm \mathbf{0 . 0 2}$ & $\mathbf{0 . 8 9} \pm \mathbf{0 . 0 1}$ & $\mathbf{0 . 9 0} \pm \mathbf{0 . 0 1}$ \\
\hline
\end{tabular}

Table 1: AUC-ROC scores on the $1000 \mathrm{~ms}$ signals.

Varying the signal length from 600 to $1400 \mathrm{~ms}$, the mean AUC-ROCs are similar $(0.71,0.90,0.89,0.91$, and 0.90) and standard deviations are all $<0.02$, except that $600 \mathrm{~ms}$ is significantly worse than each of the others $(p<0.0001)$.

\section{Discussions and Conclusions}

A multi-lead SO-BSS based method $A A C F(N, N)$ was developed to separate atrial periodic sources from surface recordings and distinguish between EBs and reentries. It is robust to Gaussian noise of up to $0.5 \mathrm{mV}$, and insensitive to the number of sources, the recording system, as well as the signal length for $\geq 800 \mathrm{~ms}$. It also outperforms $A F F \operatorname{Tr}_{2 D F}$, overcoming the difficulties for FFTbased methods in picking DF, especially for complex FFT spectrograms, as well as filters for noise removal. Its superiority over $A C F_{V 1}(\tilde{\tau})$ shows that not only the estimation of $\tau$ but also BSS are important for classifying EBs.

In this study, $K=N$ and $5 \leq N \leq 9$ were selected for $A A C F(K, N)$ due to the convergence of $K \geq 4$ in Fig. 4, but the optimal choices of $K$ and $N$ require further testing. Future development includes applying the method on different virtual patients, as well as to clinical signals to assess the correlation of EB and the ablation outcome.

In conclusion, on simulations of stable reentries and regular EBs, regardless of the heterogeneity of LA surface, our multi-lead SO-BSS-based method has shown advantages in identifying different types of periodic source in AF over the single-lead FFT analysis method. The method is easy to use, robust to Gaussian noise, and insensitive to the number of sources, signal length, as well as ECG recording systems, making it suitable for low-cost pre-ablation assessment and post-ablation management.

\section{Acknowledgments}

Funding has been received from the European Union Horizon 2020 research and Innovation programme "Personalised In-silico Cardiology (PIC)" under the Marie Sklodowska-Curie grant agreement No 764738, and the French National Research Agency (ANR-10-IAHU-04).

\section{References}

[1] Choi S, Cichocki A, Park HM, Lee SY. Blind source separation and independent component analysis: A review. Neural Inform Process Lett Rev 2005;6(1):1-57.

[2] Castells F, Rieta J, Millet J, Zarzoso V. Spatiotemporal Blind Source Separation Approach to Atrial Activity Estimation in Atrial Tachyarrhythmias. IEEE Trans Biomed Eng Feb. 2005;52(2):258-267.

[3] Llinares R, Igual J. Exploiting periodicity to extract the atrial activity in atrial arrhythmias. EURASIP J Adv Signal Process 2011;2011(1).

[4] Sameni R, Jutten C, Shamsollahi M. Multichannel electrocardiogram decomposition using periodic component analysis. IEEE Trans Biomed Eng Aug. 2008;55(8):1935-1940.

[5] Vigmond EJ, Hughes M, Plank G, Leon L. Computational tools for modeling electrical activity in cardiac tissue. J Electrocardiol Dec. 2003;36(SUPPL.):69-74. 


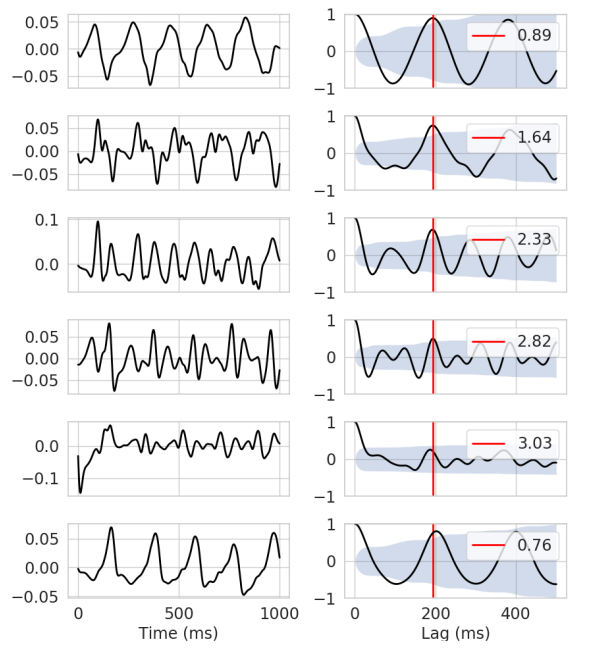

(a) Stable AF reentry with a $\mathrm{CB}$
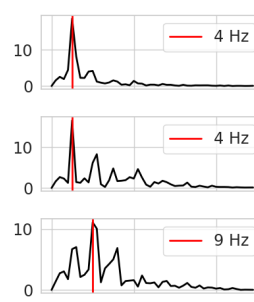

Whing
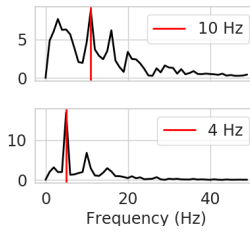
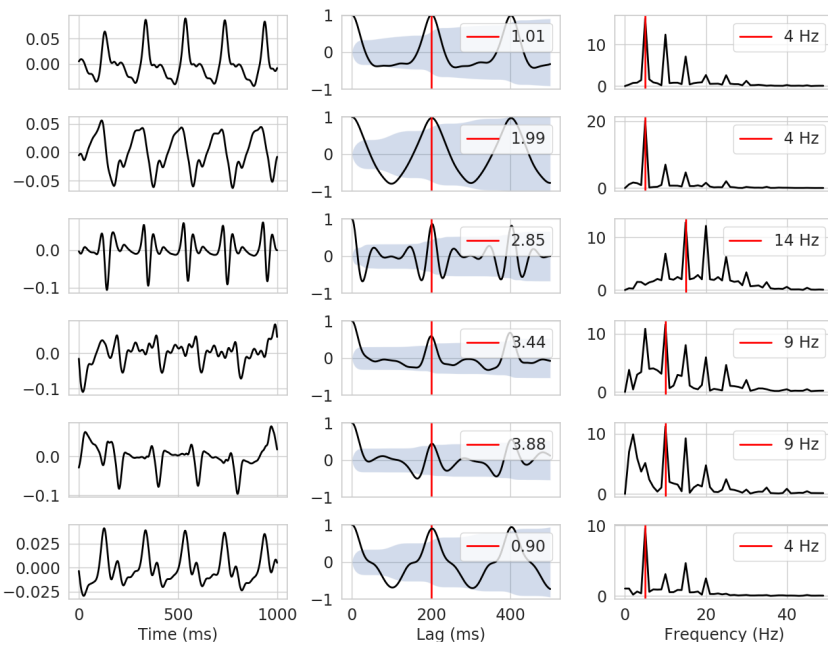

$N_{0}^{10}$
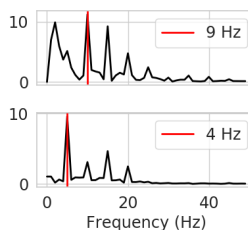

(b) EB on homogeneous LA

Fig. 3: Decomposition of two noise-free 1000ms 12-lead ECG signals into five sources using our methods. The first five rows show the top five sources as ranked by their eigenvalues, with the bottom row showing the V1 ECG, for stable AF reentries and EBs on homogeneous LA. The first column shows the amplitude $(\mathrm{mV})$ of the source. The second shows $A C F_{i}(t)$ or $A C F_{V 1}(t)$ with the lag variable $t$, where the red bar marks $\tilde{\tau}$ with labels of $A A C F(K, N)$ or $A C F_{V 1}(\tilde{\tau})$, and the shade shows the $95 \%$ confidence interval. The third is the FFT spectrogram with DF in each red bar.

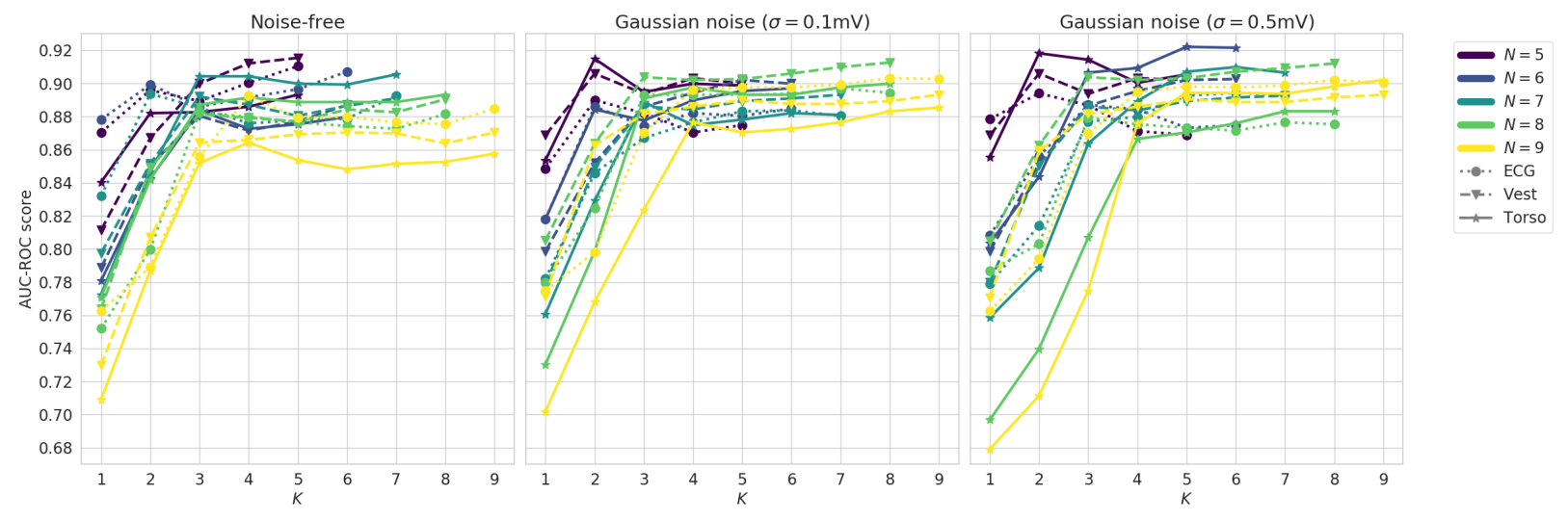

Fig. 4: AUC-ROC scores of $A A C F(K, N)$ of $1000 \mathrm{~ms}$ signals, relating to three different noise levels.

[6] Labarthe S, et al. A bilayermodel of human atria:mathematical background, construction, and assessment. Europace 2014;16(May):iv21-iv29.

[7] Roney $\mathrm{CH}$, et al. Universal atrial coordinates applied to visualisation, registration and construction of patient specific meshes. Med Image Anal July 2019;55:65-75.

[8] Kneller J, Zou R, Vigmond EJ, Wang Z, Leon LJ, Nattel S. Cholinergic Atrial Fibrillation in a Computer Model of a Two-Dimensional Sheet of Canine Atrial Cells With Realistic Ionic Properties. Circ Res May 2002;90(9).

[9] Bayer JD, et al. Acetylcholine Delays Atrial Activation to Facilitate Atrial Fibrillation. Front Physiol Sept. 2019; 10:1105.

[10] Tong L, Soon VC, Huang YF, Liu R. AMUSE: A new blind identification algorithm. IEEE Int Symp Circ S 1990; 3:1784-1787.
[11] Belouchrani A, Abed-Meraim K, Cardoso JF, Moulines E. A blind source separation technique using second-order statistics. IEEE Trans Signal Process 1997;45(2):434-444.

[12] Box GE, Jenkins GM, Reinsel GC, Ljung GM. Time series analysis: forecasting and control. John Wiley \& Sons, 2015.

[13] Alday EAP, Colman MA, Langley P, Zhang H. Novel noninvasive algorithm to identify the origins of re-entry and ectopic foci in the atria from 64-lead ECGs: A computational study. PLoS Comput Biol Mar. 2017;13(3):e1005270.

Address for correspondence:

Yingjing Feng

IHU Liryc, F-33600 Pessac-Bordeaux, France

yingjing.feng@ihu-liryc.fr 